\title{
硅烷偶联剂表面处理对 $\mathrm{LiNi}_{0.8} \mathrm{Co}_{0.15} \mathrm{Al}_{0.05} \mathrm{O}_{2}$ 结构和性能的影响
}

\author{
范广新 ${ }^{1}$, 刘泽萍 ${ }^{1}$, 闻寅 ${ }^{1}$, 刘宝忠 ${ }^{2}$ \\ (河南理工大学 1. 材料科学与工程学院; 2 . 化学化工学院, 焦作 454000)
}

摘 要: 用硅烷偶联剂加热分解的简便方法对锂离子电池正极材料 $\mathrm{LiNi}_{0.8} \mathrm{Co}_{0.15} \mathrm{Al}_{0.05} \mathrm{O}_{2}(\mathrm{NCA})$ 的表面进行处理, 利 用 XRD 结合 Rietveld 精修、SEM、TEM、DSC、EIS 和恒流充放电等方法对材料进行表征。结果显示, 硅烷偶联 剂经 $450^{\circ} \mathrm{C}$ 加热分解后得到的非晶态 $\mathrm{SiO}_{2}$ 均匀包覆在材料表面, 包覆不改变 $\mathrm{NCA}$ 的晶体结构, 但明显改善了材料 性能。在 $60^{\circ} \mathrm{C}$ 环境中, $0.2 C 、 1 C$ 下包覆材料(简写为 a-NCA) 的放电比容量分别为 $176.4 、 158.9 \mathrm{mAh} \cdot \mathrm{g}^{-1}$, 高于 $\mathrm{NCA}$ 的 $174.2 、 153.8 \mathrm{mAh} \cdot \mathrm{g}^{-1} ; 50$ 周循环后 $\mathrm{a}-\mathrm{NCA}$ 的容量保持率为 $91.4 \%$, 远高于 $\mathrm{NCA}$ 的 $86.5 \%$; 同时, 经包覆后材料 的热稳定性大幅度提高。其原因是包覆层抑制了 $\mathrm{NCA}$ 在循环过程中与电解液发生副反应, 有效降低了离子迁移的 界面膜电阻, 并抑制了晶体结构变化。

关 键 词: $\mathrm{LiNi}_{0.8} \mathrm{Co}_{0.15} \mathrm{Al}_{0.05} \mathrm{O}_{2}$; 硅烷偶联剂; 包覆; 电化学性能; 热稳定性

中图分类号: TQ152 文献标识码: A

\section{Surface Treatment on Structure and Property of $\mathrm{LiNi}_{0.8} \mathrm{Co}_{0.15} \mathrm{Al}_{0.05} \mathrm{O}_{2}$ by Silane Coupling Agent}

\author{
FAN Guang-Xin ${ }^{1}$, LIU Ze-Ping ${ }^{1}$, WEN Yin ${ }^{1}$, LIU Bao-Zhong ${ }^{2}$
}

(1. School of Materials Science and Engineering, Henan Polytechnic University, Jiaozuo 454000, China; 2. College of Chemistry and Chemical Engineering, Henan Polytechnic University, Jiaozuo 454000, China)

\begin{abstract}
Silane coupling agent was used to treat surface of cathode material $\mathrm{LiNi}_{0.8} \mathrm{Co}_{0.15} \mathrm{Al}_{0.05} \mathrm{O}_{2}$ (NCA) for lithium ion battery by a simple thermal decomposition method. Structures and properties of the materials were characterized by XRD combined with Rietveld refinement, SEM, TEM, DSC, EIS and galvanostatic charge/discharge test. The results show that amorphous $\mathrm{SiO}_{2}$ obtained by decomposition of silane coupling agent is uniformly coated on the surface of NCA whose crystal structure remain unaffected while its comprehensive properties are significantly improved especially at high temperature. In the environment of $60^{\circ} \mathrm{C}$ at $0.2 \mathrm{C}$ and $1 \mathrm{C}$ rate, the discharge capacities of the coated NCA (a-NCA) are $176.4 \mathrm{mAh} \cdot \mathrm{g}^{-1}$ and $158.9 \mathrm{mAh} \cdot \mathrm{g}^{-1}$ respectively, whereas counterparts of the pristine NCA are slightly lower and reduced to $174.2 \mathrm{mAh} \cdot \mathrm{g}^{-1}$ and $153.8 \mathrm{mAh} \cdot \mathrm{g}^{-1}$, respectively. The capacity retention of a-NCA is $91.4 \%$ while that of NCA is $86.5 \%$ after 50 charge/discharge cycles. Moreover, thermal stability of a-NCA is greatly improved compared with the uncoated material under test condition of $60^{\circ} \mathrm{C}$ in that the coating inhibits the side reaction between NCA surface and electrolyte which successfully restrains the surface film resistance and restricts variation of the crystal structure of material in process of cycling.
\end{abstract}

Key words: $\mathrm{LiNi}_{0.8} \mathrm{Co}_{0.15} \mathrm{Al}_{0.05} \mathrm{O}_{2}$; silane coupling agent; coating; electrochemical performance; thermal stability

收稿日期: 2017-09-28; 收到修改稿日期：2018-01-31

基金项目：国家自然科学基金(51671080,51471065, U1304522); 河南省高校科技创新团队(16IRTSTHN005)

National Natural Science Foundation of China (51671080, 51471065, U1304522); Program for Innovative Research Team (in Science and Technology) in the University of Henan Province (16IRTSTHN005)

作者简介: 范广新(1970-), 男, 博士, 副教授. E-mail: fangx@hpu.edu.cn

通讯作者: 刘宝忠, 教授. E-mail: bzliu@hpu.edu.cn 
随着锂离子电池在电动交通工具和储能领域的 推广应用，价格低廉、综合性能优异的正极材料已 成为该领域的研究热点之一 ${ }^{[1]}$ 。富镍层状氧化物 $\mathrm{LiNi}_{0.8} \mathrm{Co}_{0.15} \mathrm{Al}_{0.05} \mathrm{O}_{2}$ (简写为 $\mathrm{NCA}$ ) 因比容量高、热稳 定性好和循环性能优良等曾被认为最有希望替代传 统的 $\mathrm{LiCoO}_{2} 、 \mathrm{LiFePO}_{4}$ 和 $\mathrm{LiMn}_{2} \mathrm{O}_{4}$ 等正极材料。尽 管自 2013 年起 NCA 就开始应用于 Telsa Model S 电 动车, 但仍存在安全性、倍率性能不佳等问题 ${ }^{[2-3]}$ 。

表面包覆可通过改变材料表面的化学成分使材 料表面能形成一层物理保护膜, 可以有效稳定电池 中材料与电解液之间的界面, 提高材料的循环能力 和安全性能, 已成为改善材料综合性能的重要方法 之一。目前已经在正极材料的研发中得到广泛应用 ${ }^{[4]}$ 。 用 $\mathrm{TiO}_{2}{ }^{[5]} 、 \mathrm{LiCoO}_{2}{ }^{[6]} 、 \mathrm{FePO}_{4}{ }^{[7]} 、 \mathrm{Ni}_{3}\left(\mathrm{PO}_{4}\right)_{2}{ }^{\left[{ }^{[8]}\right.}$ 和 $\mathrm{SnO}_{2}{ }^{[9]}$ 等作为包覆层都能不同程度地提升 $\mathrm{NCA}$ 的性能。

在锂离子电池中, 电解液中残留的痕量 $\mathrm{H}_{2} \mathrm{O}$ 往 往会和 $\mathrm{LiPF}_{6}$ 反应生成 $\mathrm{HF}$, 而 $\mathrm{HF}$ 和电极材料反应 将导致电池循环性能的恶化 ${ }^{[4]}$ 。研究发现, 正极材料 表面存在的 $\mathrm{Si}$ 元素能有效中和 $\mathrm{HF}^{[10]}$ 。因此, 来源 广泛、价格低廉、热稳定性良好的 $\mathrm{SiO}_{2}$ 可以用作改 善正极材料性能的包覆物质。到目前为止, 已经有 用 $\mathrm{SiO}_{2}$ 包覆 $\mathrm{LiNi}_{0.5} \mathrm{Mn}_{1.5} \mathrm{O}_{4}{ }^{[11]} 、 \mathrm{LiNi}_{0.8} \mathrm{Co}_{0.1} \mathrm{Mn}_{0.1} \mathrm{O}_{2}{ }^{[12]}$ 、 $\mathrm{LiNi}_{0.915} \mathrm{Co}_{0.075} \mathrm{Al}_{0.01} \mathrm{O}_{2}{ }^{[13]} 、 \mathrm{LiNi}_{0.6} \mathrm{Co}_{0.2} \mathrm{Mn}_{0.2} \mathrm{O}_{2}{ }^{[14]}$ 和 $\mathrm{LiFePO}_{4}{ }^{[15]}$ 等的公开报道。

在包覆 $\mathrm{SiO}_{2}$ 的方法中, 机械混合法操作简单, 却难以将 $\mathrm{SiO}_{2}$ 均匀包覆在基体材料表面, 溶胶一凝 胶法、原子沉积法和化学反应法等虽包覆效果较好, 但操作相对复杂、不宜工业化大规模生产 ${ }^{[10-15]}$ 。硅 烷偶联剂是具有特殊结构的低分子有机硅化合物, 具有无毒、成本低等特点, 广泛应用于无机粉体材 料的表面处理 ${ }^{[16]}$ 。在锂离子电池领域, 它可以在 $\mathrm{LiCr}_{x} \mathrm{Mn}_{2-x} \mathrm{O}_{4}$ 表面生成稳定的富硅层, 实现材料改 性 ${ }^{[17]}$ 。

本文以硅烷偶联剂为原料、利用它可溶于水的 特点, 用简易的工艺对 NCA 进行表面处理, 并系 统分析该处理过程对材料结构和性能的影响, 以期 为 NCA 提供一种操作简便、效果良好的改性方法。

\section{1 实验方法}

\subsection{NCA 的制备及表面处理过程}

按照摩尔比 $\mathrm{Li}: \mathrm{M}(\mathrm{M}=\mathrm{Ni} 、 \mathrm{Co} 、 \mathrm{Al})=1.05: 1$ 的 比例, 将 $\mathrm{Li}_{2} \mathrm{CO}_{3}$ 与 $\mathrm{Ni}_{0.85} \mathrm{Co}_{0.1} \mathrm{Al}_{0.5}(\mathrm{OH})_{2}$ 前驱体充分研 磨, 混合均匀的粉末在空气气氛下 $650^{\circ} \mathrm{C}$ 保温 $5 \mathrm{~h}$ 后再 $780^{\circ} \mathrm{C}$ 煅烧 $15 \mathrm{~h}$, 得到的正极材料 $\mathrm{LiNi}_{0.8} \mathrm{Co}_{0.15} \mathrm{Al}_{0.05} \mathrm{O}_{2}$
(标记为 NCA)。

用硅烷偶联剂对 NCA 表面进行处理的过程如 下: 分别将 $0.05 、 0.1 、 0.2 、 0.3 \mathrm{~g}$ 硅烷偶联剂 KH550(化 学式 $\left.\mathrm{NH}_{2} \mathrm{CH}_{2} \mathrm{CH}_{2} \mathrm{CH}_{2} \mathrm{Si}\left(\mathrm{OC}_{2} \mathrm{H}_{5}\right)_{3}\right)$ 加入到 $50 \mathrm{~mL}$ 酒精 和水(质量比为 $10: 1$ ) 的混合液中; 恒温 $50^{\circ} \mathrm{C}$ 磁力摚 拌 $20 \mathrm{~min}$, 再分别加入 $\mathrm{NCA}$ 粉末 $10.0 \mathrm{~g}$, 继续搅拌 $20 \mathrm{~min}$ 后, 将混合物放入烘箱中干燥; 经研磨、置 于空气气氛箱式炉中, 在 $450^{\circ} \mathrm{C}$ 保温 $5 \mathrm{~h}$, 得到表面 处理后的 $\mathrm{LiNi}_{0.8} \mathrm{Co}_{0.15} \mathrm{Al}_{0.05} \mathrm{O}_{2}$ 正极材料。为行文简 洁, 本文仅选加入 $0.2 \mathrm{~g}$ 硅烷偶联剂、综合性能最佳 的材料, 标记为 a-NCA。

\section{2 材料表征}

使用日本理学配有石墨弯晶单色器的 Smart-lab 型 X 射线衍射仪采集数据, 管压 $40 \mathrm{kV}$, 管流 $200 \mathrm{~mA}$, $\mathrm{CuK} \alpha$ 靶辐射, $\lambda=0.15406 \mathrm{~nm}$, 连续扫描速度 $2 \% \mathrm{~min}$, 扫描范围 $2 \theta=15^{\circ} \sim 85^{\circ}$; 微结构和晶粒尺寸用 MDI Jade 软件 Rietveld 精修获得。采用德国蔡司 Merlin Compact 型场发射扫描电镜(SEM) 获得材料的颗粒 形貌和尺寸, 并装配牛津仪器能谱仪(EDS)对材料 的成分进行分析。用日本 JEM-2100 透射电子显微 镜(TEM)观察材料表面的处理情况。在美国 Ta 公司 差示扫描量热仪 Q100 和同步热分析仪 Q600 上进行 差示扫描量热(DSC)和 TG-DTA 热分析。

\section{3 电化学性能测试}

将正极材料、聚偏氟乙烯(PVDF)和乙炔黑按质 量比 $8: 1: 1$ 充分混合, 以 $N$-甲基吡咯烷酮(NMP) 为溶剂, 配制成浆料均匀涂抹在铝䈃上, 在 $100^{\circ} \mathrm{C}$ 真空箱中干燥 $12 \mathrm{~h}$ 制成正极片; 以金属锂片为负 极、美国 Celgard2300 为隔膜、 $1 \mathrm{~mol} / \mathrm{L} \mathrm{LiPF}_{6}$ 的碳 酸乙烯酯/碳酸二乙酯(EC/DEC)溶液为电解液, 在 充满氩气的手套箱中组装成 CR2016 型纽扣电池。 采用深圳新威电池测试系统(CT3008W-5 V $10 \mathrm{~mA}$ ) 进行恒流充放电测试, 测试过程分别在室温 $25^{\circ} \mathrm{C}$ (RT)和高温 $60^{\circ} \mathrm{C}(\mathrm{HT})$ 环境下进行, 充放电截止电压 为 4.3 2.75 V。使用美国普林斯顿 PARSTAT 2273 型电化学工作站进行交流阻抗测试, 电极电位的正 弦波振幅限制为 $5 \mathrm{mV}$, 测试频率范围为 $0.01 \mathrm{~Hz}$ $100 \mathrm{kHz}$ 。

\section{2 结果与讨论}

\section{1 硅烷偶联剂的热处理分析}

图 1(a)是硅烷偶联剂在空气气氛中从室温到 $450^{\circ} \mathrm{C}$ 的 TG-DTA 曲线。DTA 曲线显示, 在 $241.8^{\circ} \mathrm{C}$ 附近出现一个较强的放热峰, 主要对应硅烷偶联剂 



图 1 硅烷偶联剂 KH550 在空气气氛中的 TG-DTA 曲线(a) 及在 $450^{\circ} \mathrm{C}$ 保温 $5 \mathrm{~h}$ 热分解产物的 XRD 图谱(b)

Fig. 1 (a) TG-DTA curves of the KH550 silane coupling agent in air and (b) XRD pattern for thermal decomposition product of the silane coupling agent after heating at $450^{\circ} \mathrm{C}$ for $5 \mathrm{~h}$

的氧化 ${ }^{[18]} ; \mathrm{TG}$ 曲线显示, 加热温度达到 $270^{\circ} \mathrm{C}$ 后, 随着温度升高产物的质量不再变化，表明在 $270^{\circ} \mathrm{C}$ 以上能够保证硅烷偶联剂热分解反应进行完全。在 分解产物的 XRD 图谱(图 1(b))中, $2 \theta=22.8^{\circ}$ 处较宽的 衍射峰与文献 ${ }^{[15,19]}$ 报道相似，可知产物是非晶态的 $\mathrm{SiO}_{2}$ 。

\section{2 硅烷偶联剂处理对 NCA 晶体结构的影响}

图 2 为采用硅烷偶联剂处理前、后 NCA 的 XRD 图谱, 由图 2(a)可知, 材料没有明显变化, 各样品均 具有六方晶系 $\alpha-\mathrm{NaFeO}_{2}$ 结构, 属于 $\mathrm{R}-3 \mathrm{~m}$ 空间群。 放大后的 XRD 图谱(图 2(b))中(006)/(012)、(018)/ (110)衍射峰有明显的分裂, 说明材料有较理想的层 状结构 ${ }^{[10-11]}$ 。在 XRD 图谱中未识别到 $\mathrm{SiO}_{2}$ 的特征 峰, 这主要由于硅烷高温分解后生成的 $\mathrm{SiO}_{2}$ 为非晶 态、且含量也较低, 所以其衍射峰的强度相对于 NCA 较弱。对 NCA 和 a-NCA XRD 数据进行 Rietveld 结 构精修的结果(如图 3、表 1)显示, 二者的结构参数 $(a 、 c 、 c / a 、 \operatorname{FWHM}(003) 、 \operatorname{FWHM}(104))$ 均相近, 表明 $\mathrm{SiO}_{2}$ 未进入 $\mathrm{NCA}$ 晶格, 没有对其晶体结构产生影响。
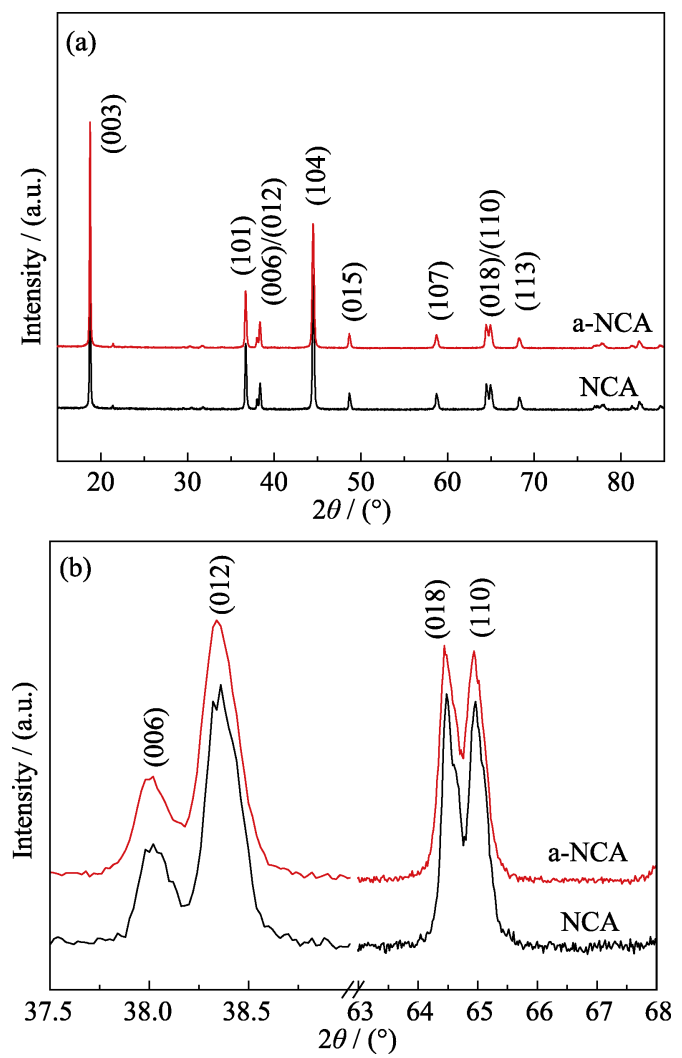

图 $2 \mathrm{NCA}$ 和 a-NCA 的 XRD 图谱(a)及局部放大图谱(b) Fig. 2 (a) XRD patterns for NCA and a-NCA, and (b) their enlarged XRD patterns.

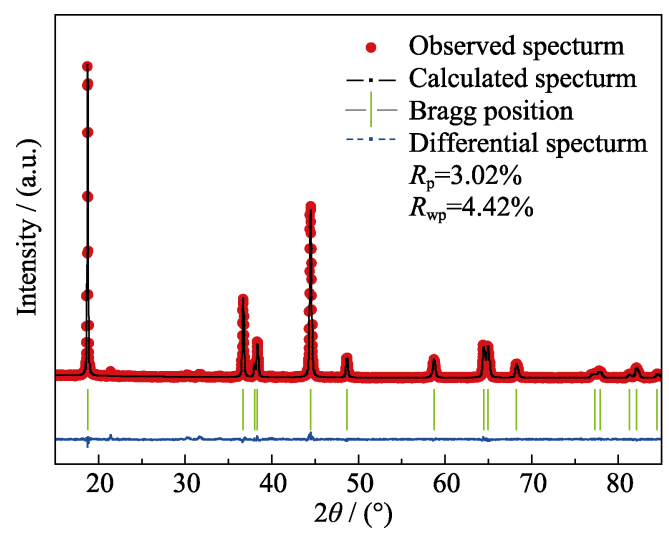

图 3 a-NCA 的 XRD Rietveld 结构精修图

Fig. 3 XRD Rietveld refinement of a-NCA

表 1 NCA 和 a-NCA 的结构参数

Table 1 Structural parameters of NCA and a-NCA

\begin{tabular}{cccccc}
\hline \multirow{2}{*}{ Sample } & \multirow{2}{*}{$a / \mathrm{nm}$} & \multirow{2}{*}{$c / \mathrm{nm}$} & \multirow{2}{*}{$c / a$} & \multicolumn{2}{c}{$\mathrm{FWHM} /\left(^{\circ}\right)$} \\
\cline { 5 - 7 } & & & & $(003)$ & $(104)$ \\
\hline NCA & 0.2869 & 1.4193 & 4.948 & 0.130 & 0.225 \\
$\mathrm{a}-\mathrm{NCA}$ & 0.2869 & 1.4200 & 4.949 & 0.131 & 0.228 \\
\hline
\end{tabular}

\section{3 硅烷偶联剂处理对 NCA 宏观形貌的影响}

图 4(a) (d)分别展示了在不同放大倍数下 NCA 和 a-NCA 的 SEM 照片。由图 4(a)和(c)可见, 各样品 均由一次粒子团聚形成的球形二次颗粒组成, 粒径分 

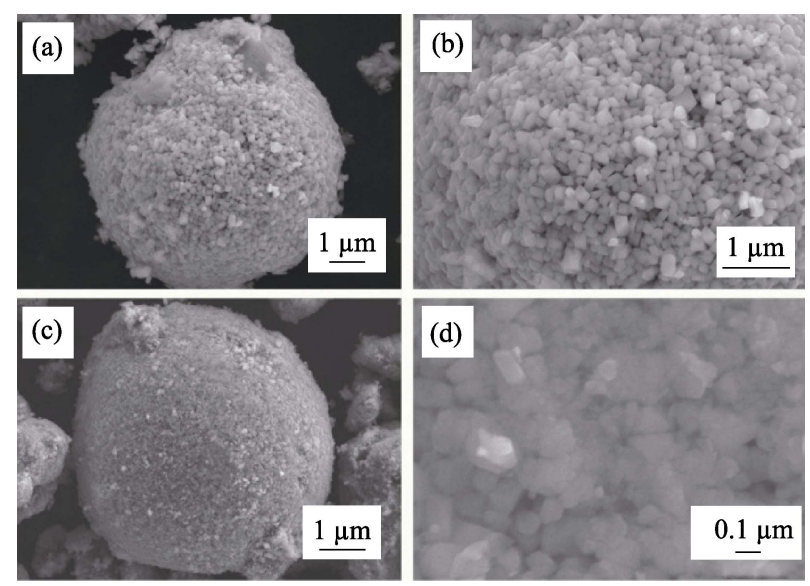

图 $4 \mathrm{NCA}(\mathrm{a}) \sim(\mathrm{b})$ 和 a-NCA (c) (d) 的 SEM 照片

Fig. 4 SEM images of $\operatorname{NCA}(a, b)$ and a-NCA $(c, d)$

布均在 8 12 $\mu \mathrm{m}$ 之间, 说明用硅烷偶联剂表面处理 对 NCA 二次颗粒的形貌和尺寸影响不大。但从图 4(b)和(d)能够明显地看出, NCA 样品的二次颗粒表 面粗楉, 一次粒子边界清晰; 而 a-NCA 二次颗粒的 表面变得相对平滑，一次粒子界面模糊，说明硅烷 经高温处理后, 包覆层包裹在 NCA 的表面。对图 4(d) 进行的 EDS 面扫描能谱分析(如图 5 所示)可知, $\mathrm{Ni} 、 \mathrm{Co} 、 \mathrm{Al}$ 和 $\mathrm{Si}$ 元素都分布均匀。用 TEM 对 a-NCA 表面观察的结果如图 6 所示, 结合硅烷高温炦烧后 的 XRD 图谱(图 1(b)) 可知, 非晶态 $\mathrm{SiO}_{2}$ 包覆在 NCA 颗粒表面, 这将有利于阻挡电解液与 NCA 的直接 接触, 抑制电解液在循环过程中产生的 HF 对 NCA 侵蚀, 从而改善材料的电化学性能 ${ }^{[10-15]}$ 。

\section{4 硅烷偶联剂处理对 NCA 电化学性能的影响}

图 7(a) 是 NCA 和 a-NCA 在室温和高温下首次 充放电曲线 (电流密度为 $0.1 C$ )。它们具有相似的充 放电特征, 但差别也较明显。从充电曲线上看, 电压 平台在室温较高、且在各温度下 a-NCA 的平台更高; 在放电曲线上，二者在高温下的电压平台基本相同， 而室温下 a-NCA 的平台略低。与图 7(a)对应的微分 容量曲线(图 7(b))显示, $\mathrm{NCA}$ 和 a-NCA 均在 3.65 3.85 V 电压范围内出现一对氧化还原峰, 对应 着 $\mathrm{Ni}^{2+} / \mathrm{Ni}^{4+}$ 的氧化还原反应 ${ }^{[10-11]}$; 该反应峰对的电 位差能够反映电极的极化, 其差值越大, 电极极化 越强 ${ }^{[10]}$; 通过分析各样品 $\mathrm{Ni}^{2+} / \mathrm{Ni}^{4+}$ 氧化还原峰对的 差值(表 2)可知, 包覆材料的差值均较小, 表明包覆 $\mathrm{SiO}_{2}$ 有利于降低 NCA 电极材料的极化现象; 且高 温时该差值由 NCA 的 $0.05 \mathrm{~V}$ 降到 a-NCA 的 $0.01 \mathrm{~V}$, 说明在高温时硅烷偶联剂处理 NCA 对降低材料的 极化现象更有优势。另外，在不同温度下, a-NCA 的 首次放电比容量均略低(表 2, 在高温下这种差别甚 微), 主要是由于用硅烷偶联剂处理后生成非电化

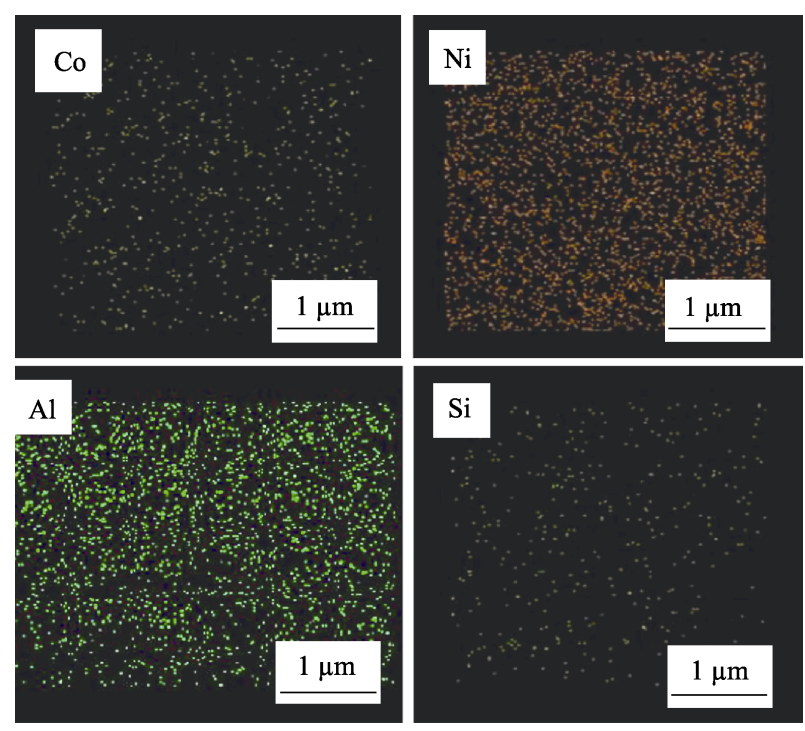

图 $5 \mathrm{a}-\mathrm{NCA}$ 的 EDS 能谱图

Fig. 5 EDS mapping of a-NCA

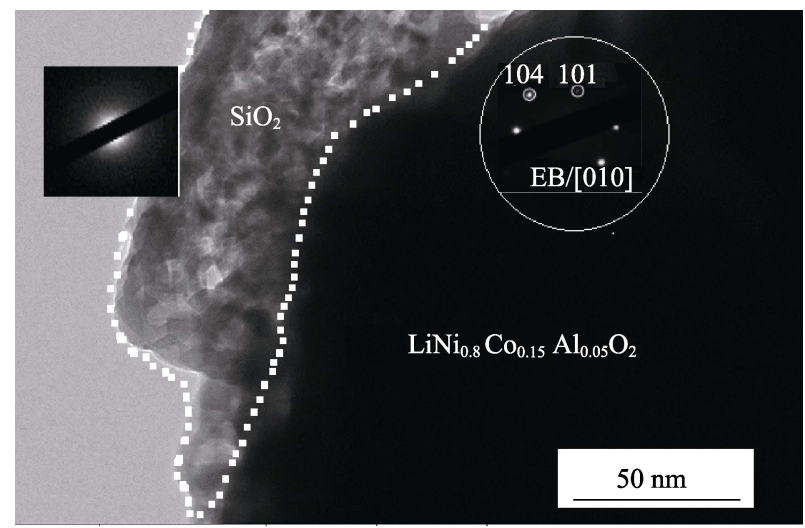

图 6 a-NCA 的 TEM 照片

Fig. 6 TEM image of a-NCA

学活性的 $\mathrm{SiO}_{2}$ 包覆层降低了电极材料中活性物质 的比例; 此外, 各样品的放电比容量在高温时比在 室温时更高(a-NCA 的差别更明显)。

$\mathrm{NCA}$ 和 a-NCA 在不同温度下的倍率和循环性 能如图 8 所示。随着电流密度的增大, 材料的放电 比容量降低。在室温 $0.2 C$ 和 $1 C$ 电流密度下, $\mathrm{NCA}$ 的放电比容量较大，而在高温时情况相反(表 2)。同 时发现，二者在室温、 $1 C$ 下的放电比容量分别为 $0.1 C$ 的 $85.1 \%$ 和 $85.2 \%$, 几乎相同; 但在高温, $1 C$ 下 的放电比容量分别为 $0.1 C$ 的 $83.2 \%$ 和 $86.7 \%$, 表明 用硅烷偶联剂处理 NCA 也有利于改善高温下材料 的倍率性能。在 50 周循环内观察到, 表面处理可明 显改善材料的循环性能, 不论在室温还是在高温条 件下, a-NCA 的循环性能都优于 NCA。这是因为循 环过程中电解液里产生的 HF 会侵蚀电极材料表面, $\mathrm{SiO}_{2}$ 包覆层能够减少电解液与电极材料颗粒表面的 直接接触，抑制电解液对材料的腐蚀，从而提高材 料的循环性能 ${ }^{[12-13,15]}$ 。尽管在高温下各样品的容量 

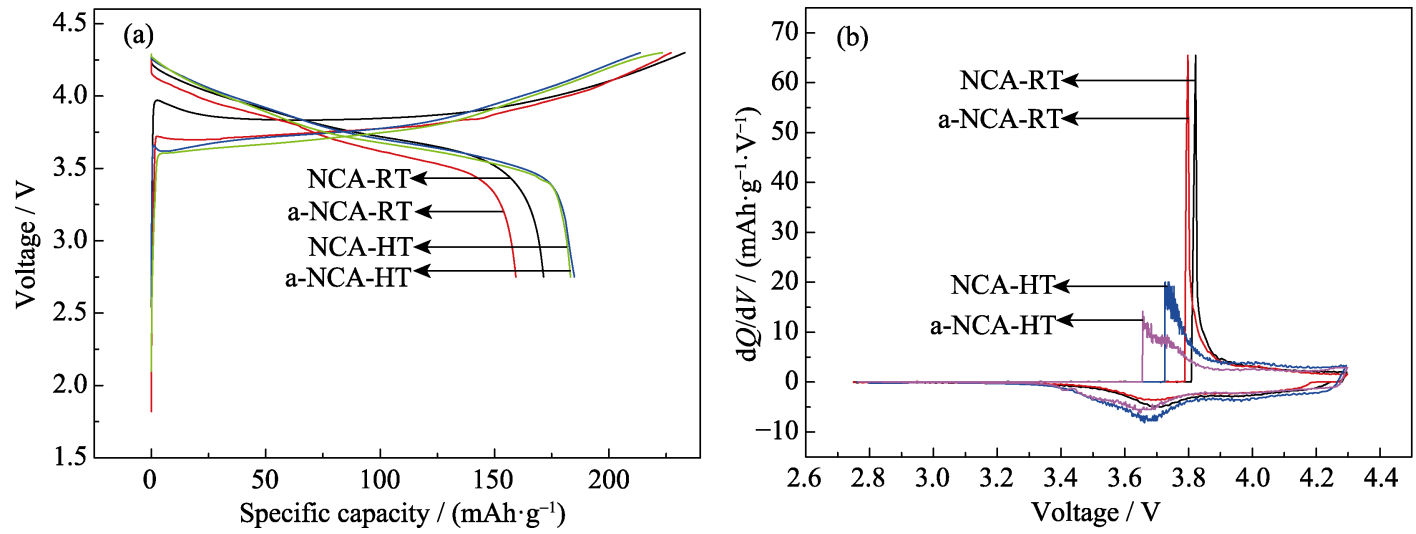

图 7 NCA 和 a-NCA 的首次充放电曲线 (a)及对应的微分容量曲线(b)

Fig. 7 (a) Initial charge-discharge curves and (b) the corresponding differential capacity $(\mathrm{d} Q / \mathrm{d} V)$ of NCA and a-NCA

表 2 NCA 和 a-NCA 的电化学性能

Table 2 Electrochemical performances of NCA and a-NCA

\begin{tabular}{cccccc}
\hline Sample & $0.1 C /\left(\mathrm{mAh} \cdot \mathrm{g}^{-1}\right)$ & $0.2 C /\left(\mathrm{mAh} \cdot \mathrm{g}^{-1}\right)$ & $1 C /\left(\mathrm{mAh} \cdot \mathrm{g}^{-1}\right)$ & Difference values of $\left(\mathrm{Ni}^{2+} / \mathrm{Ni}^{4+}\right) / \mathrm{V}$ & Capacity retention \\
\hline NCA-RT & 171.5 & 164.7 & 146.0 & 0.11 & $94.7 \%$ \\
a-NCA-RT & 164.6 & 153.8 & 140.2 & 0.10 & $96.0 \%$ \\
NCA-HT & 184.9 & 174.2 & 153.8 & 0.05 & $86.5 \%$ \\
a-NCA-HT & 183.2 & 176.4 & 158.9 & 0.01 & $91.4 \%$ \\
\hline
\end{tabular}

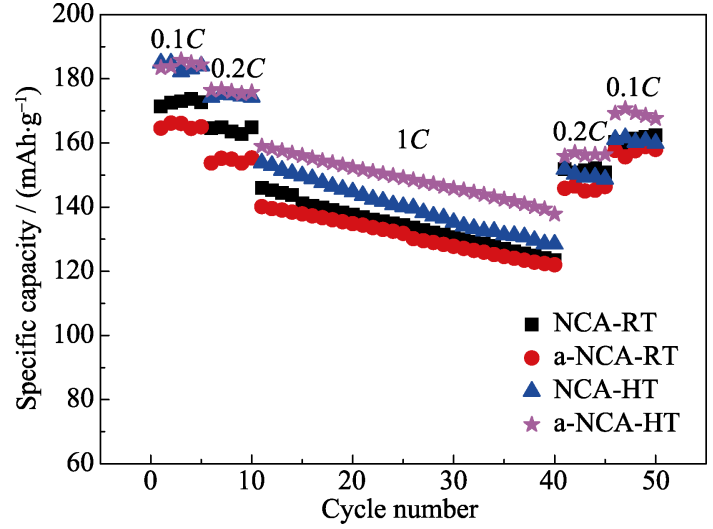

图 8 NCA 和 a-NCA 的倍率和循环性能

Fig. 8 Rate capacity and cyclic ability of NCA and a-NCA

保持率均略低, 但经硅烷偶联剂表面处理后材料的 循环性能提高更明显, 经过高温 50 周循环后, 容量

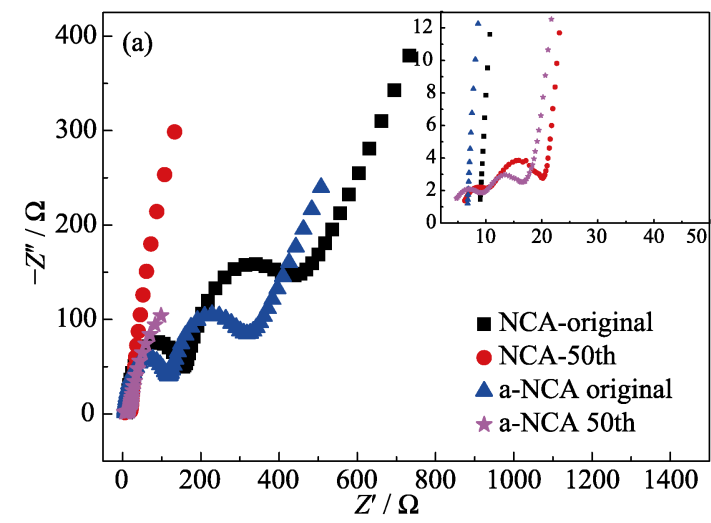

仍高达 $167.4 \mathrm{mAh} / \mathrm{g}$ 。值得说明的是，尽管本研究所 使用的硅烷偶联剂种类、具体包覆工艺等仍需优化, 但 其改善 NCA 电化学性能的效果明显优于直接用 $\mathrm{SiO}_{2}$

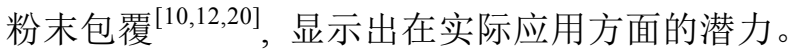

图 9(a)和(b)分别为 NCA 和 a-NCA 在不同温度 下的交流阻抗 Nyquist 图，(b)中的插图为其相应的 等效电路。图中: 高频区的半圆弧代表电解液和电 极表面的极化反应形成的 SEI 膜阻抗 $\left(R_{\mathrm{f}}\right)$; 中频区的 半圆弧代表电荷转移电阻 $\left(R_{\mathrm{ct}}\right)$; 低频区斜线反映的 是 $\mathrm{Li}^{+}$在固体电极材料中的扩散能力, 即 Warburg 阻 抗 $\left(W_{\mathrm{o}}\right)^{[12,20]}$ 。另外, $R_{\mathrm{S}}$ 代表电池的体相电阻(如电解 液、隔膜、电极金属极片元件的电阻)。用 Zview 软 件对等效电路进行拟合后的结果(表 3)显示，初始 状态下, a-NCA 与 $\mathrm{NCA}$ 相比, $R_{\mathrm{s}} 、 R_{\mathrm{f}}$ 和 $R_{\mathrm{ct}}$ 均较小、

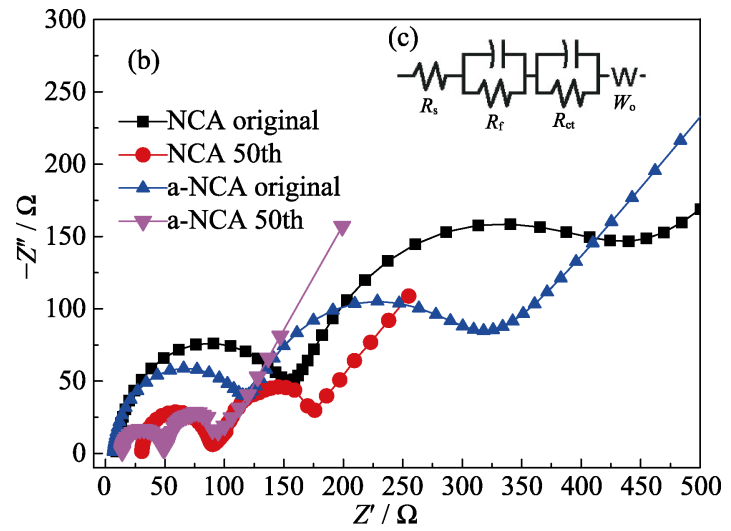

图 9 NCA 和 a-NCA 在(a)室温和(b)高温的交流阻抗图, (b)中的插图为等效电路

Fig. 9 EIS plots of NCA and a-NCA at (a) room temperature and (b) high temperature with inset in (b) showing the corresponding equivalent circuit diagram 
表 3 NCA 和 a-NCA 循环前后的交流阻抗值

Table 3 Electrochemical AC impedance values of NCA and a-NCA

\begin{tabular}{cccccc}
\hline Sample & Condition & $R_{\mathrm{S}} / \Omega$ & $R_{\mathrm{f}} / \Omega$ & $R_{\mathrm{ct}} / \Omega$ & $W_{\mathrm{o}} /\left(\times 10^{-3}, \Omega\right)$ \\
\hline \multirow{3}{*}{ NCA } & Original & 8.6 & 136.4 & 211.9 & 2.0 \\
& RT-50th & 7.5 & 8.3 & 126.1 & 2.8 \\
& HT-50th & 7.9 & 55.7 & 114.2 & 7.5 \\
& Original & 6.5 & 106.0 & 156.6 & 3.1 \\
a-NCA & RT-50th & 4.5 & 7.4 & 92.1 & 4.9 \\
& HT-50th & 6.2 & 21.4 & 75.5 & 8.6 \\
\hline
\end{tabular}

而 $W_{\mathrm{o}}$ 较大, 表明用硅烷偶联剂处理后使电池的体 相电阻、电极的 SEI 膜阻抗和电荷转移电阻都减小, $\mathrm{Li}^{+}$在电极和电解液界面的扩散能力提高。经 50 周 循环后, 各阻抗也都呈现降低趋势, 并且 a-NCA 的 各阻抗减小更显著。值得注意的是, a-NCA 的初始 $R_{\mathrm{f}}$ 就明显低于 $\mathrm{NCA}$, 经高温 50 周循环后, 前者的 $R_{\mathrm{f}}$ 进一步降低到后者的二分之一以下, 表明 $\mathrm{SiO}_{2}$ 包覆 层有效地保护了 $\mathrm{NCA}$ 的表面不受电解液中 $\mathrm{HF}$ 的侵 蚀; 同时, 虽然 $\mathrm{SiO}_{2}$ 包覆层本身导电性差, 不利于 电子和 $\mathrm{Li}^{+}$在 $\mathrm{NCA}$ 和电解液间的迁移, 但提高了电 极和电解液界面 $\mathrm{Li}^{+}$的扩散能力, 所以 $\mathrm{a}-\mathrm{NCA}$ 的 $R_{\mathrm{ct}}$ 反而低于 $\mathrm{NCA}$, 说明包覆层限制电解液腐蚀的保护 作用在影响 $R_{\mathrm{ct}}$ 的因素中起主导作用。由此可见, a-NCA 倍率性能和循环性能的提高主要得益于 $\mathrm{SiO}_{2}$ 包覆层对 NCA 表面的保护, 降低了阻抗, 提高了 $\mathrm{Li}^{+}$在电极和电解液界面的扩散能力。

\section{5 硅烷偶联剂处理对 NCA 热稳定性的影响}

经 50 周循环后将电池充电至 $4.30 \mathrm{~V}$, 对正极材 料进行 DSC 分析, 结果如图 10 所示。NCA 和 a-NCA 在 $200 \sim 250^{\circ} \mathrm{C}$ 温度范围内均存在一个较明显的放热 峰, 已知该放热峰主要由(充电态)高活性的含氧物 质(例如 $\mathrm{O}^{2-} 、 \mathrm{O}_{2}^{2-} 、 \mathrm{O}_{2}$ 等)与电解液反应放热产生 ${ }^{[21]}$ 。 在经过同样条件的循环后, a-NCA 的放热峰温度都 得到提高, 表明硅烷偶联剂处理可抑制充电态正极 材料分解释放氧, 提高与电解液的反应温度, 从而 提高材料的热稳定性能。

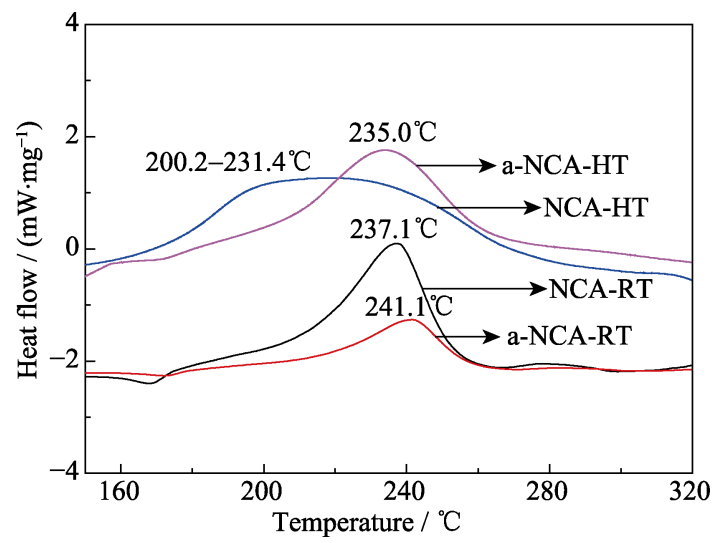

图 $10 \mathrm{NCA}$ 和 a-NCA 的 DSC 曲线

Fig. 10 DSC curves of NCA and a-NCA

\section{6 硅烷偶联剂处理对 NCA 循环过程中晶体 结构的影响}

为进一步探讨硅烷处理对 NCA 正极材料循环 过程中晶体结构的影响, 图 11 给出了 $\mathrm{NCA}$ 和 a-NCA 在循环前及不同温度下 50 次循环后的 XRD 局部放大图谱。由图 11(a) (b)可知, 在不同温度下 经过 50 次循环后, 各样品仍保持原来的 $\alpha-\mathrm{NaFeO}_{2}$ 型层状结构, 衍射峰也未发生明显宽化, 该峰向低 角度偏移, 标志着晶胞参数 $c$ 值增大, 这是因为在循 环过程中, $\mathrm{Li}^{+}$从晶体结构中脱出后不能全部可逆嵌 入, 使 $\mathrm{Li}$ 层中 $\mathrm{Li}^{+}$减少, 导致相邻氧层间静电斥力 增大的结果 ${ }^{[22-23]}$ 。显然, a-NCA 的(003)衍射峰偏移 较小, 意味着 a-NCA 在循环过程中 $\mathrm{Li}^{+}$脱/嵌的可逆 性较好。分析两种样品循环前后晶体结构参数(表 4)可以看出, 循环后二者的晶胞参数 $a 、 V$ 均减小, $c$ 值增大; 但 a-NCA 的晶胞参数变化较小, 在高温的 情况下, 二者的差别更为明显, 以 $a$ 为例, 高温循环 后, a-NCA 的 $\triangle a / a$ (循环 50 周后 $a$ 与初始状态 $a$ 的 差值 $\Delta a$ 与初始 $a$ 的比值)值不足 NCA 的二分之一。 结果表明, 用硅烷处理 NCA 可有效抑制材料在循环 过程中晶体结构的变化, 在高温环境下表现更为突出。
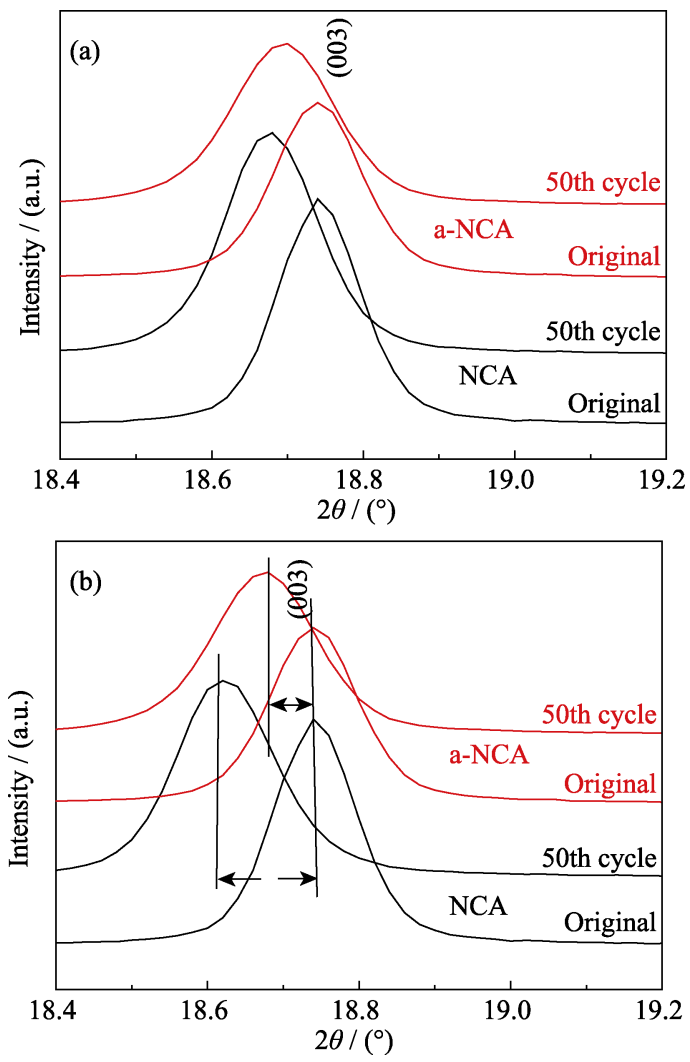

图 $11 \mathrm{NCA}$ 和 a-NCA 在室温(a)和高温(b)循环前后(003)峰 的 XRD 图谱

Fig. 11 XRD patterns of (003) peak in original and the $50^{\text {th }}$ cycle sample for NCA and a-NCA at room temperature (a) and high temperature (b) 


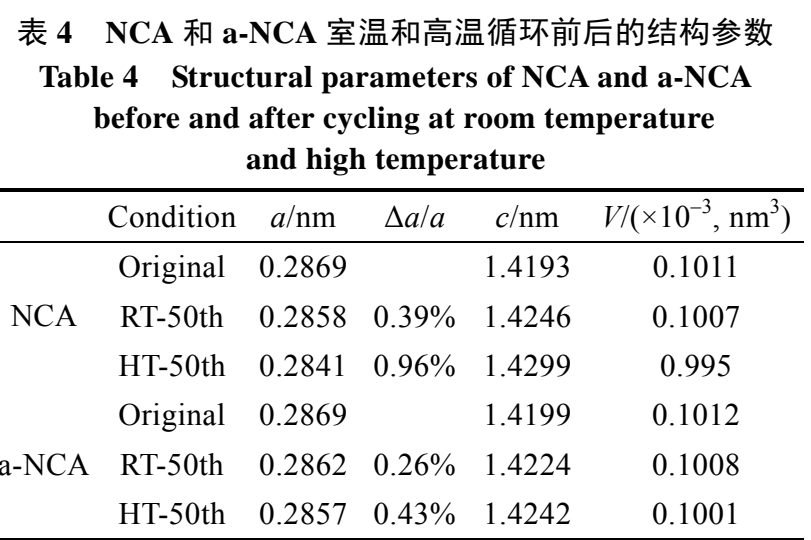

\section{3 结论}

1) 用硅烷偶联剂经高温 $\left(450^{\circ} \mathrm{C}\right)$ 加热可以在正 极材料 $\mathrm{LiNi}_{0.8} \mathrm{Co}_{0.15} \mathrm{Al}_{0.05} \mathrm{O}_{2}$ 表面包覆 $\mathrm{SiO}_{2}$, 该工艺 具有简便、可行的优点。 $\mathrm{SiO}_{2}$ 包覆层为非晶态, 不 影响基底材料的晶体结构。

2) $\mathrm{SiO}_{2}$ 包覆后, 材料的放电比容量略有降低, 但极化现象、循环性能、倍率性能、热稳定性有明 显改善, 尤其在高温 $60^{\circ} \mathrm{C}$ 测试条件下, 综合性能大 幅提高, 显示出 $\mathrm{SiO}_{2}$ 包覆后的材料在高温工作环境 下具有实际应用的潜力。

3) 综合性能的提高得益于 $\mathrm{SiO}_{2}$ 包覆层降低了 电极材料的界面膜电阻, 提高了电极和电解液界面 上 $\mathrm{Li}^{+}$的扩散能力, 有效抑制了材料在循环过程中 晶体结构的变化。

\section{参考文献:}

[1] NITTA N, WU F, LEE J T, et al. Li-ion battery materials: present and future. Mater. Today, 2015, 18(5): 252-264.

[2] TRAN H Y, TÄUBERT C, WOHLFAHRT-MEHRENS M. Influence of the technical process parameters on structural mechanical and electrochemical properties of $\mathrm{LiNi}_{0.8} \mathrm{Co}_{0.15} \mathrm{Al}_{0.05} \mathrm{O}_{2}$ based electrodes - a review. Prog. Solid State Ch., 2014, 42(4): 118 -127.

[3] WANG H, LAI C, XIAO Y, et al. A new lithium-ion battery with $\mathrm{LiNi}_{0.8} \mathrm{Co}_{0.15} \mathrm{Al}_{0.05} \mathrm{O}_{2}$ cathode and lithium pre-doping hard carbon anode. Mater. Lett., 2015, 160: 250-254.

[4] ZUO D, TIAN G, LI X, et al. Recent progress in surface coating of cathode materials for lithium ion secondary batteries. J. Alloys Comp., 2017, 706: 24-40.

[5] XU Y, LI X, WANG Z, et al. Structure and electrochemical performance of $\mathrm{TiO}_{2}$-coated $\mathrm{LiNi}_{0.8} \mathrm{Co}_{0.15} \mathrm{Al}_{0.05} \mathrm{O}_{2}$ cathode materials. Mater. Lett., 2015, 143: 151-154.

[6] LIU W, HU G, DU K, et al. Surface coating of $\mathrm{LiNi}_{0.8} \mathrm{Co}_{0.15} \mathrm{Al}_{0.05} \mathrm{O}_{2}$ with $\mathrm{LiCoO}_{2}$ by a molten salt method. Surf. Coat. Tech., 2013, 216: 267-272.
[7] HUANG B, LI X, WANG Z, et al. A facile process for coating amorphous $\mathrm{FePO}_{4}$ onto $\mathrm{LiNi}_{0.8} \mathrm{Co}_{0.15} \mathrm{Al}_{0.05} \mathrm{O}_{2}$ and the effects on its electrochemical properties. Mater. Lett., 2014, 131: 210-213.

[8] LEE D J, SCROSATI B, SUN Y K. $\quad \mathrm{Ni}_{3}\left(\mathrm{PO}_{4}\right)_{2}$-coated $\mathrm{LiNi}_{0.8} \mathrm{Co}_{0.15} \mathrm{Al}_{0.05} \mathrm{O}_{2}$ lithium battery electrode with improved cycling performance at $55^{\circ} \mathrm{C}$. J. Power Sources, 2011, 196(18): 7742-7746.

[9] HE X, DU C, SHEN B, et al. Electronically conductive Sb-doped $\mathrm{SnO}_{2}$ nanoparticles coated $\mathrm{LiNi}_{0.8} \mathrm{Co}_{0.15} \mathrm{Al}_{0.05} \mathrm{O}_{2}$ cathode material with enhanced electrochemical properties for Li-ion batteries. Electrochim. Acta, 2017, 236: 273-279.

[10] CHEN C, TAO T, WANG Q, et al. High-performance lithium ion batteries using $\mathrm{SiO}_{2}$-coated $\mathrm{LiNi}_{0.5} \mathrm{Co}_{0.2} \mathrm{Mn}_{0.3} \mathrm{O}_{2}$ microspheres as cathodes. J. Alloys Comp., 2017, 709: 708-716.

[11] FAN Y, WANG J, TANG Z, et al. Effects of the nanostructured $\mathrm{SiO}_{2}$ coating on the performance of $\mathrm{LiNi}_{0.5} \mathrm{Mn}_{1.5} \mathrm{O}_{4}$ cathode materials for high voltage Li-ion batteries. Electrochim. Acta, 2007, 52(11): 3870-3875.

[12] LIANG L, HU G, JIANG F, et al. Electrochemical behaviours of $\mathrm{SiO}_{2}$-coated $\mathrm{LiNi}_{0.8} \mathrm{Co}_{0.1} \mathrm{Mn}_{0.1} \mathrm{O}_{2}$ cathode materials by a novel modification method. J. Alloys Comp., 2016, 657: 570-581.

[13] ZHOU P, ZHANG Z, MENG $\mathrm{H}$, et al. $\mathrm{SiO}_{2}$-coated $\mathrm{LiNi}_{0.915} \mathrm{Co}_{0.075} \mathrm{Al}_{0.01} \mathrm{O}_{2}$ cathode material for rechargeable Li-ion batteries. Nanoscale, 2016, 8(46): 19263-19269.

[14] CHO W, KIM S M, SONG J H, et al. Improved electrochemical and thermal properties of nickel rich $\mathrm{LiNi}_{0.6} \mathrm{Co}_{0.2} \mathrm{Mn}_{0.2} \mathrm{O}_{2}$ cathode materials by $\mathrm{SiO}_{2}$ coating. J. Power Sources, 2015, 282: 45-50.

[15] LI Y, ZHAO S. Electrochemical performance of $\mathrm{SiO}_{2}$-coated $\mathrm{LiFePO}_{4}$ cathode materials for lithium ion battery. J. Alloys Comp., 2011, 509(3): 957-960.

[16] MALLAKPOUR S, MADANI M. A review of current coupling agents for modification of metal oxide nanoparticles. Prog. Org. Coat., 2015, 86: 194-207.

[17] LIU HUAN-MIN, HUANG KE-LONG, XUE JIAN-JUN, et al. Study on the performance of $\mathrm{LiCr}_{x} \mathrm{Mn}_{2-x} \mathrm{O}_{4}$ by surface treatment with silane coupling agent. Battery Bimonthly, 2004, 34(6): 403405 .

[18] 王雪明. 硅烷偶联剂在金属预处理及有机涂层中的应用研究. 济南: 山东大学硕士学位论文, 2005.

[19] HUANG WEI, CAO XUE-JUAN, ZHU HONG-ZHOU, et al. Research on physic adsorption and photocatalytic activity of $\mathrm{TiO}_{2} / \mathrm{SiO}_{2}$ with different molar content of $\mathrm{SiO}_{2}$. J. Wuhan Univ. Technol., 2015, 37(9): 25-31.

[20] CHO Y, CHO J. Significant improvement of $\mathrm{LiNi}_{0.8} \mathrm{Co}_{0.15} \mathrm{Al}_{0.05} \mathrm{O}_{2}$ cathodes at $60^{\circ} \mathrm{C}$ by $\mathrm{SiO}_{2}$ dry coating for Li-ion batteries. J. Electrochem. Soc., 2010, 157(6): A625-A629.

[21] SHI Y, ZHANG M, QIAN D, et al. Ultrathin $\mathrm{Al}_{2} \mathrm{O}_{3}$ coatings for improved cycling performance and thermal stability of $\mathrm{LiNi}_{0.5} \mathrm{Co}_{0.2} \mathrm{Mn}_{0.3} \mathrm{O}_{2}$ cathode material. Electrochim. Acta, 2016, 203: 154-161.

[22] YIN S C, RHO Y H, SWAINSON I, et al. X-ray/neutron diffraction and electrochemical studies of lithium de/re-intercalation in $\mathrm{Li}_{1-x} \mathrm{Co}_{1 / 3} \mathrm{Ni}_{1 / 3} \mathrm{Mn}_{1 / 3} \mathrm{O}_{2} \quad(x=0 \rightarrow 1)$. Chem. Mater, 2006, 18(7): 1901-1910.

[23] LIN C K, REN Y, AMINE K, et al. In situ high-energy X-ray diffraction to study overcharge abuse of 18650-size lithium-ion battery. J. Power Sources, 2013, 230: 32-37. 